\title{
Study on the Voltage Regulation Device at the End of the Low Voltage Line
}

\author{
XIE Shengli ${ }^{1}$, Zhang Xiaodong ${ }^{1}$, Liu Liping ${ }^{1}$, Ning Bo $^{2}$, Song Huixin ${ }^{1}$ \\ ${ }^{1}$ Skill Training Center of the State Grid Jibei Electric Power Company Limited, Baoding 071003, \\ China \\ ${ }^{2}$ Measurement Center of Marketing Department, State Grid Jibei Electric Power Company Limited, \\ Beijing
}

Keywords: Low voltage line; the multi-tapped transformer; voltage adjusting device

\begin{abstract}
In view of the phenomenon of the voltage fluctuation with the load at the users' end to some degree caused by the power investment restrictions, the scattered residents, long lines, and the large line loss in the economically backward areas, a method of the voltage adjusting device composed of a multi-tapped transformer and power electronic switches to compensate the voltage is put forward in this paper, and a control system design method of the voltage adjusting device is presented. And the experiment results show that the method is effective and feasible.
\end{abstract}

\section{Introduction}

With the end of the rural power grid construction and renovation, the safety and health of rural power grids greatly improved, the overall voltage quality also improved greatly, which has provided a strong electric guarantee for rural economic development and new rural construction ${ }^{[1]}$. But the limited funding of the rural power transformation and the inherent scattered distribution of the rural power grid led to the incomplete transformation of the rural power grid, especially in the remote mountainous villages and economically backward areas, the scattered residents, long lines, and the large line loss caused the phenomenon of the voltage fluctuation with the load at the users' end to some degree, which seriously affected people's quality of life, reduced the electrical life, and brought the power fire hazards and caused discontent among the residents.

The existing voltage regulating schemes usually have two types. One is a parallel capacitor is installed at the end of the line for reactive power compensation, but the booster effect this scheme is not obvious ${ }^{[2-3]}$. The other is to adjust the transformer tap at the head end of the line, but the effect does not meet the requirements at the end of the line and the scheme needs to re-design lines and install the terminal transformer, so the investment is too large, not economic.

In view of the above problems, this paper proposes to install an automatic voltage adjusting device at the end of the line; the device can automatically adjust the size of the output voltage with the change of the input voltage, so that the output voltage is maintained within a certain range so as to meet user demands.

\section{Working Principle of the Voltage Adjusting Device}

The voltage adjusting device is mainly composed of the following three parts:

(1) The multi-tapped transformer. The multi-tapped transformer consists of a primary side tap and many secondary side taps. Taking the line rated input voltage of $220 \mathrm{~V}$ as an example, 7 secondary side taps are set respectively as: 95\%, 97.5\%, 100\%, 102.5\%, 105\%, 107.5\%, and $110 \%$. The rated current can be designed as $200 \mathrm{~mA}$, and the transformer capacity is designed as $20 \mathrm{VA}$. 


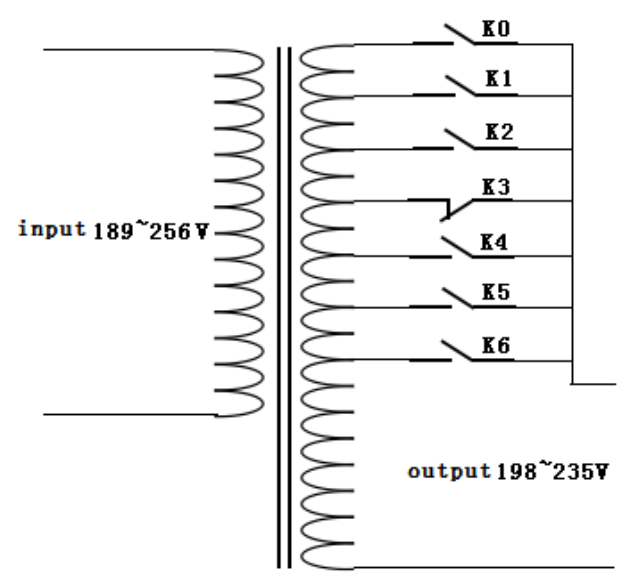

Fig.1 the multi-tapped transfer

(2)The tap switch circuit

The tap switch circuit consists of 7 power electronic switches, wherein, 1 switch is in the closed state, connecting the $100 \%$ transformer tap, the remaining 6 switches are in the turn-off state, connecting the other 6 transformer taps.

(3)The core control circuit

The high-speed ARM processor system is used to collect the voltage at the output end of the transformer; when the voltage is at the allowable range, the $100 \%$ tap is used, and the output voltage is directly transformed; when the voltage is higher than the upper limit or less than the lower limit, it will automatically adjust the action of the relay, switch the tap position, and the output voltage is controlled within the permissible range.

Figure 2 is the control circuit diagram of the voltage adjusting device, in Figure 2, a side tap is connected to the input end of line voltage; very secondary tap corresponds to a different coil number; the head end of many electronic switch elements are respectively connected with the secondary taps of the transformer, and the terminal of the electron switch element is connected with the load user. A voltage detection device detects the circuit output voltage all the time; and then feedback voltage real-time signal to the microprocessor in the adjustable control unit; the microprocessor judges whether the voltage at both line ends deviates from its normal value or not through a comparison operation, if the voltage is lower or higher than the normal value, the microprocessor will send a signal to indicate the adjustment increase voltage; the electronic switch control device sends the switch signal to control the electronic switch elements (K0 K6) to achieve the purpose of adjusting circuit output voltage.

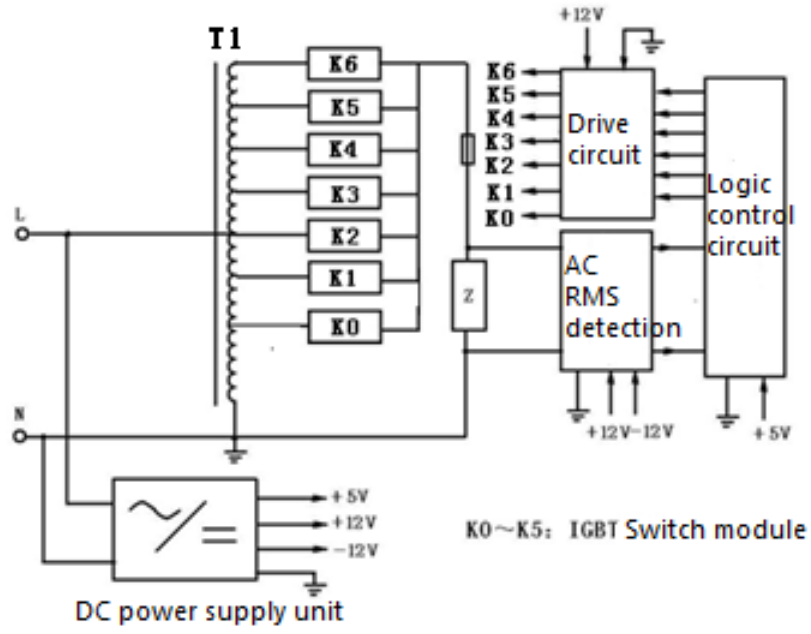

Fig.2 the control circuit diagram of the voltage adjusting device 


\section{Control Circuit Design of the Voltage Adjusting Device}

The control circuit of the voltage adjusting device is shown in Figure 2; it consists of a DC power supply unit, a RMS detection unit of the output voltage, a logic control circuit unit, a driving unit of the power electronic switch.

\section{Schematic diagram of DC power supply.}

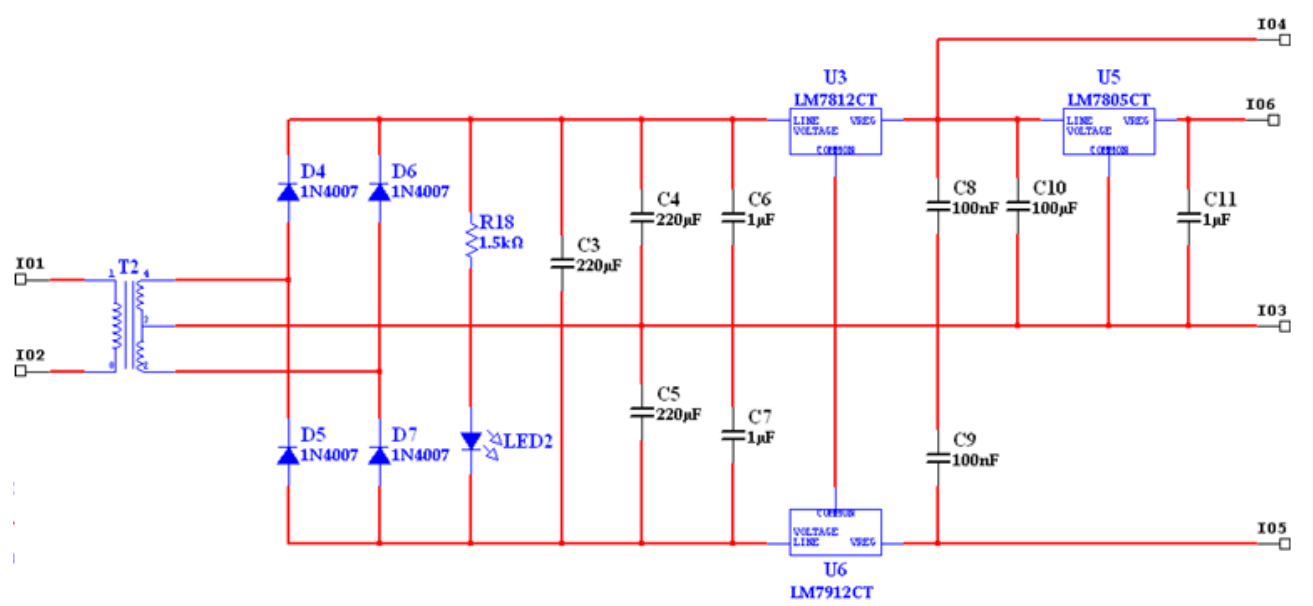

Fig.3 the circuit diagram of the DC power supply unit

Fig. 3 is the circuit diagram of the DC power supply unit. In this diagram circuit, the voltage is taken from the transmission lines of I01, I02, and it changes into a single secondary-side 0.05 transformer via it and is connected with ratio for the of the and rectifier diode connected the rectifier diode, after the voltage is rectified and filtered, the C3 voltage is about $30 \mathrm{~V}$, dual power voltage at the input end is about $15 \mathrm{~V}$ or so, and then use 7805 , 7812 and 7912 to get $+5 \mathrm{~V}$ and $\pm 12 \mathrm{~V}$ DC voltage. Taking into account the input voltage may be reduced to $160 \sim 170 \mathrm{~V}$, while the maximum input voltage of the three -terminal regulator is $36 \mathrm{~V}$, so a single secondary-side transformer ratio can selected to be 0.08 to 0.1 , the full transformation ratio can be 0.2 . When the 0.08 single ratio is selected, and the input AC voltage changes from $240 \mathrm{~V}$ to $170 \mathrm{~V}$, and the 7812 and the 7912 input voltage is $26 \mathrm{~V} \sim 18.5$, which is more reliable and safe.

In addition, the current of the three -terminal regulator is set to be $1 \mathrm{~A}$, although the control circuit current is very small, the addition of the margin is a good choice.

The detection circuit of RMS output voltage.Fig. 4 is the detection circuit of the RMS output voltage

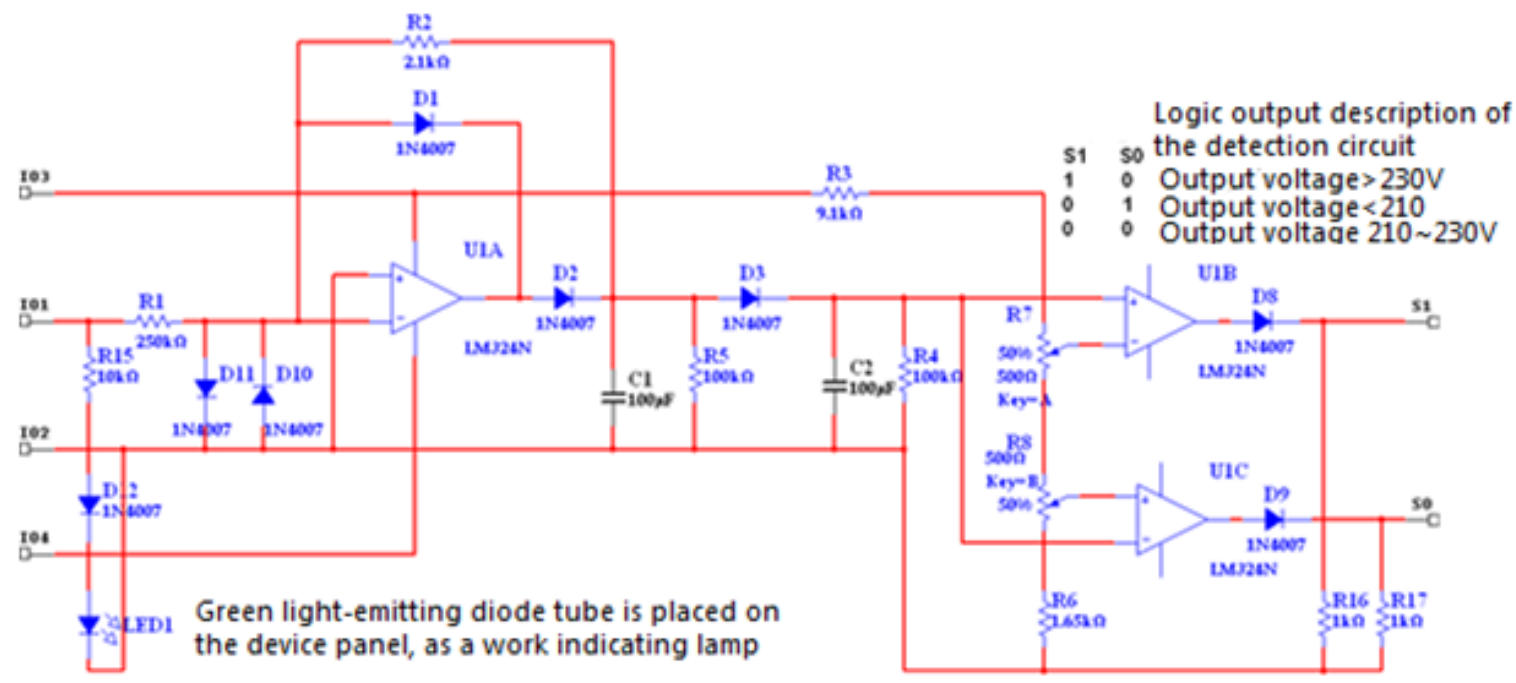

Fig. 4 the detection circuit of the RMS output voltage 
The circuit consists of a four op amp LM324 and a diode, wherein, U1A and peripheral devices constitute the active filter circuit, when the input AC voltage is $220 \mathrm{~V}$, the DC output voltage after filtering is approximately $2.2 \mathrm{~V}$, and the voltage is approximately proportional to the input AC voltage. C1 and R5, according to the parameters shown above, the circuit can start after 0.2s. R7 and R8 divide the voltage of the $+12 \mathrm{~V}$ DC power supply, the upper and lower limit reference voltage corresponding to $230 \mathrm{~V}$ and $210 \mathrm{~V}$ is respectively obtained; and then use the two voltage comparators of $\mathrm{U} 1 \mathrm{~B}, \mathrm{U} 1 \mathrm{C}$ to compare with the output voltage of the active filter circuit, the control signal of S1 and S0 are obtained. The voltage logic judgment of S1 and S0 is shown in the following figure.

\subsection{The logic control circuit}

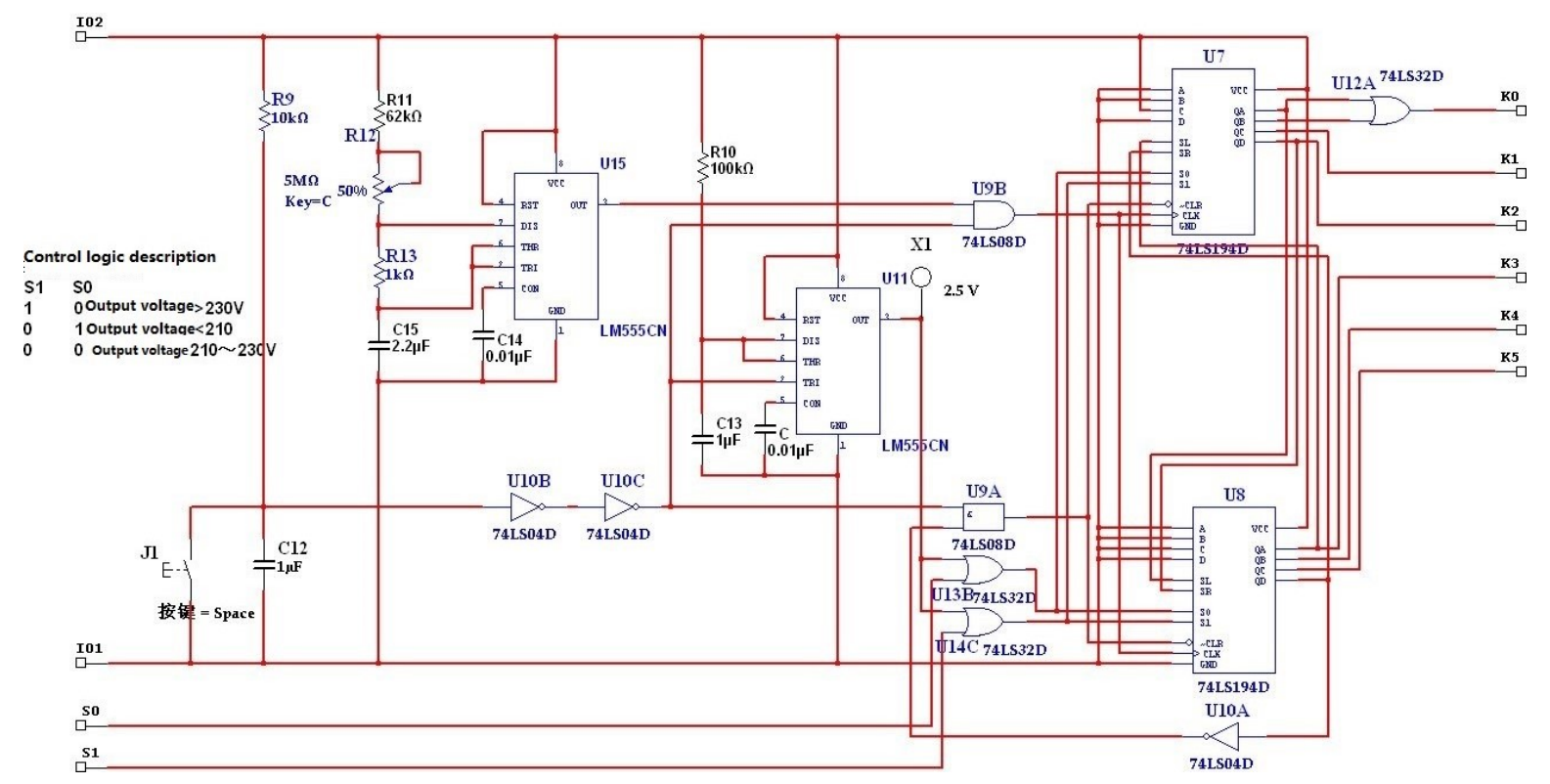

Fig.5 the logic control circuit

In Figure 5:The logic control circuit; the role of each component are respectively as follows:

(2) 15: A multi harmonic oscillator, its frequency can be adjusted, the output width is constant, and the period is adjustable in $0.1 \mathrm{~s} \sim 8 \mathrm{~s}$, it controls the time of IGBT module switching.

(2) $\mathrm{J} 1$ is an external reset button, installed on the shell of the device.

(3) 11 constitutes a monostable trigger, and the delay time is $0.1 \mathrm{~s}$, which is used for register set to ensure the working of the K1IGBT module when it is automatically powered, at the same time, it shields K0, K2 K5 modules, so as to prevent the circulation at the instant starting.

(4) 74194 or 74198 constitute the switching circuits of the K0 K5 IGBT modules.

Function description:

At the instant after power on, the voltage of C12 capacitance is zero, after the drive of two inverter plastic triggers, a low level trigger unit is obtained; at the same time, through the U9A, 74194 or 74198 will be reset by the unit and the gate circuit. Make the output K0 K5 be zero, IGBT does not work.

The monostable trigger outputs a high level after around $0.1 \mathrm{~s}$ of the trigger delay, make the input ends of 741974 or 74198 be 1 at the same time. During this period, U10C generates the jumping voltage pulse to trigger 741974 or 74198 , make the K1 be 1 , and the start is completed.

(1) Work process:

When $\mathrm{S} 0=1, \mathrm{~S} 1=0$, indicating that the output voltage is less than the lower limit; it is needed to boost, the circuit will work in the direction of $\mathrm{K} 2 \rightarrow \mathrm{K} 3 \rightarrow \mathrm{K} 4 \rightarrow \mathrm{K} 5$, respectively make the $\mathrm{K} 2 \sim$ K5IGBT module work to boost. When $\mathrm{S} 0=0$, $\mathrm{S} 1=1$, indicating that the output voltage is greater than the upper limit; it is needed to step down, the circuit will work in the direction of $\mathrm{K} 2 \rightarrow \mathrm{K} 1 \rightarrow \mathrm{K} 1$, make the K1 IGBT module work for two times for step-down. U12A or gate in the circuit is set to prevent over-voltage malfunction. 
When $\mathrm{S} 0=0, \mathrm{~S} 1=0$, indicating that the output voltage is between the upper and lower limits, it is no need to adjust, keep the last IGBT working.

After K5 works, if the voltage is still below the lower limit, or after K0 works, the voltage is still greater than the upper limit, the circuit will automatically reset, all signals of K0 K5 are 0, IGBT is cut, constituting double protection for overvoltage and undervoltage.

After the circuit is protected, it is needed to press the reset button of $\mathrm{J} 1$, and then the circuit can work again.

In addition, a normally open inching button and two light-emitting diodes are mounted on AC regulator panel.

The status of the two light-emitting diodes is as follows:

(1)Red light-emitting tube is lit, indicating the electric supply is on; Green light-emitting tube is lit, indicating that the regulator works.

(2) Red light-emitting tube is lit, and the green light-emitting tube is off, indicating AC voltage stabilizer in the over-voltage protection status.

(3) If the red and green light-emitting tubes are off, indicating the electric supply is not powered.

\subsection{Output module of the power electronic switch driving signal}

The output module of the driving signal is mainly used to control the power electronic switch elements, and its control circuit is as shown in Figure 6.

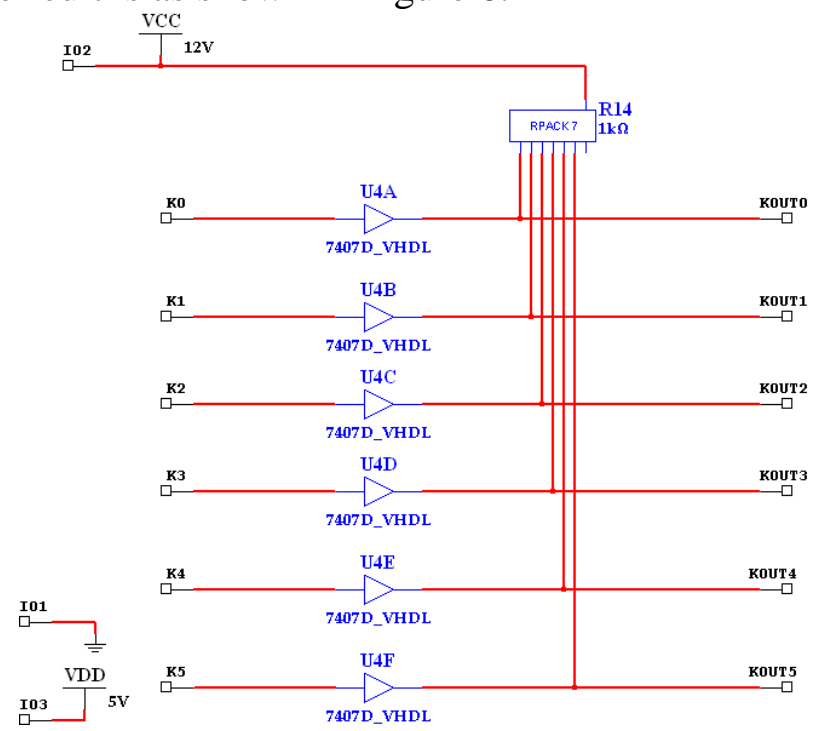

Fig.6 the output circuit of the driving signals

In this circuit, can use 7407 or 5407 TTL open collector six normal phase high pressure buffer driver can be used to control the voltage conversion of R14 to be 1-7, 1-8 exclusion, 7-7 exclusion

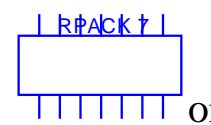
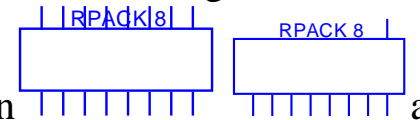
resistor.

\section{Test Results Analysis of the Voltage Adjusting Device}

According to the design of the above voltage adjustment device, the output voltage is designed to be $220 \mathrm{~V}(210 \mathrm{~V}$ to $230 \mathrm{~V}$ ), rated capacity has three kinds of $3 \mathrm{kVA}, 5 \mathrm{kVA}, 10 \mathrm{kVA}$; the input voltage is set to be 5 automatically adjustment stalls: $150 \mathrm{~V}, 165 \mathrm{~V}, 180 \mathrm{~V}, 195 \mathrm{~V}, 210 \mathrm{~V}$; the effectively regulating range of the input voltage is $140 \mathrm{~V} \sim 225 \mathrm{~V}$, and output voltage within this range is qualified.

Taking into account the device is mainly for remote mountainous areas or economically underdeveloped areas, and its user loads is mainly for the lighting facilities, so the output voltage can be set to be $220 \mathrm{~V}$, the input voltage can be set to be from $140 \mathrm{~V}$ gradually boost to $225 \mathrm{~V}$, the 
relationship of input voltage and output voltage is shown in Figure 7. The output voltage can be well maintained at about $220 \mathrm{~V}$ through the voltage adjusting device, especially when the voltage drop is large, the adjusting effect is particularly evident.

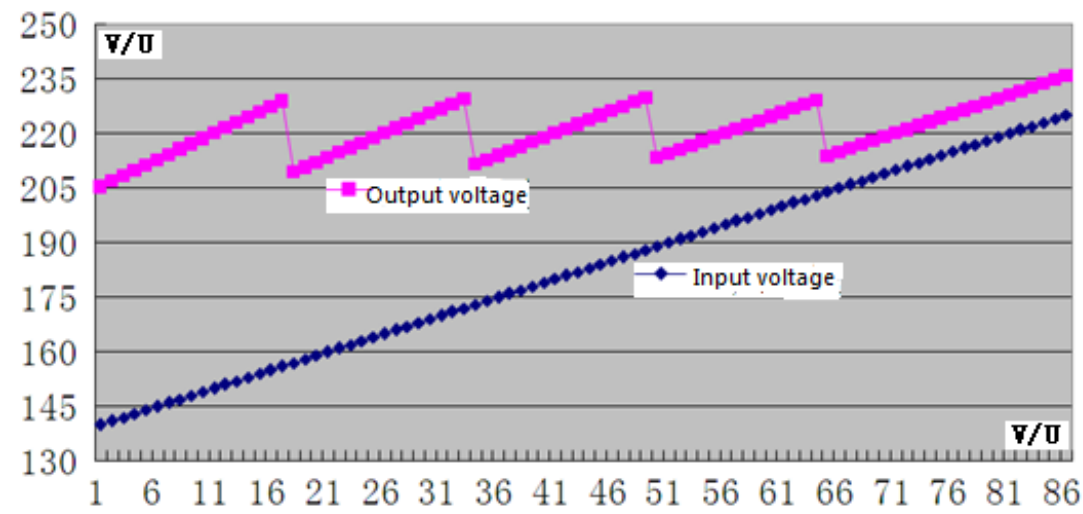

Fig.7 the relationship between the input/output voltages of the voltage adjusting device

\section{Conclusion}

Through the operation of the voltage adjusting device and economic technical analysis, it is concluded that the device can improve the voltage quality of the rural residents who are far away from the power supply source and it only needs a small amount of money in the investment, and it is an effective way to solve the problem of low voltage at the end of the rural power grid with the characteristics of less investment, quick effect, easy installation and operation maintenance.

\section{References:}

[1] Ye Min, Approach to Solve the Terminal Low Voltage of Rural Power Grid [J]. Rural Electrification, 2007.4: 51 52

[2] Bai Xuefeng, Li Pei. Development of Experiment Device in the Single-phase AC of Voltage Regulating Circuit [J].Experimental Technology and Management, 2006.7: 46 48

[3] Liu Xingping. A Novel Single-phase AC Voltage Regulation Circuit Controlled by Using Pulse Width Modulator SG3525 [J]. Machine Tool Electrical Appliances, 2002.2: 43 44

[4] CHEN Hai-rong,ZHANG Jing,TU Qing-rui. Negative Voltage Compensating Control of Voltage Source Converter Based HVDC System Under Unbalanced Grid Conditions [J]. High Voltage Engineering , 2011.10: 23 24

[5] FENG Lei,LI Xiao-fan. Study on One Kind of Voltage Compensation Means Based on Phase Shifter Control [J].Telecom Power Technologies , 2007.04: 26 29 